\title{
Competencias semióticas para la alfabetización multimodal en la asignatura de español de secundaria en Costa Rica
}

Semiotic competencies for multimodal literacy in Spanish in Costa Rica's high schools.

\author{
Competências semióticas para a alfabetização multimodal na \\ disciplina de espanhol do ensino médio na Costa Rica.
}

\author{
Carol Flores Solano \\ Universidad de Costa Rica \\ Turrialba, Costa Rica \\ carol.flores@ucr.ac.cr \\ (DD https://orcid.org/0000-0003-3771-6339
}

Recibido - Received - Recebido: 07/07/2020 Corregido - Revised - Revisado: 16/10/2020 Aceptado - Accepted - Aprovado: 02/11/2020

DOl: https://doi.org/10.22458/ie.v22i33.3037

URL: https://revistas.uned.ac.cr/index.php/innovaciones/article/view/3037

\begin{abstract}
Resumen: En respuesta a los objetivos de aprendizaje relacionados con textos multimodales, en el plan de estudios de español costarricense (MEP, 2017), y la escasez de propuestas didácticas a nivel nacional para su tratamiento pedagógico en secundaria (Flores, 2019), este artículo tiene como objetivo proponer una serie de competencias semióticas (Tobón, 2005) para facilitar los procesos de enseñanza y aprendizaje de la multimodalidad en el aula. Estas competencias se han formulado desde el enfoque sociocognitivo (Tobón, 2005), los niveles de creación de significados en textos multimodales (Kress y Van Leeuwen, 2001; Kress, 2010) y la taxonomía de Bloom para las habilidades cognitivas (Talbert, 2019). Como resultado, se plantean como procesos complejos, para la aproximación, caracterización, análisis, explicación, evaluación y producción de textos multimodales, en interacciones y contextos socioculturales específicos. Con base en la descripción de estas competencias, el artículo sostiene la necesidad de incorporar una dimensión semiótica dentro de la competencia comunicativa en el plan de estudios de español (MEP, 2017) y brindar un apoyo teórico - metodológico al profesorado para orientar la trasposición de los objetivos del currículo nacional, relacionados con textos multimodales, al salón de clases. Para concluir, sugiere vínculos entre esta propuesta y estudios sobre la comprensión lectora de textos multimodales, a fin de actualizar los procesos didácticos y curriculares nacionales, según el desarrollo de las alfabetizaciones múltiples a nivel internacional (Anstey y Bull 2006; Cope y Kalantzis, 2009b; Blanchard y Farstrup 2011; Cooper et al., 2013; Reid and Comber 2004; Walsh 2010).
\end{abstract}

Palabras clave: competencias, multimodalidad, enseñanza multimodal, alfabetizaciones múltiples, semiología. 
Abstract: In response to the learning goals related to multimodal texts in the Costa Rican Spanish study program (MEP, 2017), and the lack of didactic proposals for its pedagogical treatment at a national level in secondary schools (Flores, 2019), this article's purpose is to offer a series of semiotic competencies (Tobón, 2005) that will help in facilitating the multimodal teaching-learning process in the classroom. These competencies feed on the sociocognitive approach (Tobón, 2005), and the levels of creation of meaning in multimodal texts (Kress and Van Leeuwen, 2001; Kress, 2010) as well as on Bloom's taxonomy for cognitive abilities (Talbert, 2019). As a result, they are presented as complex processes, for the approach, characterization, analysis, explanation, evaluation, and elaboration of multimodal texts, in specific sociocultural contexts and interactions. Based on the description of these competencies, the article condones the need to incorporate a semiotic dimension within the communicative competencies in the Spanish curriculum (MEP, 2017). It seeks to provide theoretical-methodological support to teachers to help them integrate the national curriculum's objectives related to multimodal texts to the classroom. On a final note, this proposal offers a suggestion of studies regarding reading comprehension of multimodal texts, in order to update the national didactic and curricular processes, according to the development of multiple literacies at an international level (Anstey and Bull 2006; Cope and Kalantzis, 2009b; Blanchard and Farstrup 2011; Cooper et al., 2013; Reid and Comber 2004; Walsh 2010).

Keywords: Competencies, multimodality, multimodal teaching, multiple literacies, semiotics

Resumo: Em resposta aos objetivos de aprendizagem relacionados com textos multimodais, no currículo espanhol da Costa Rica (MEP, 2017), e à escassez de propostas didáticas em nível nacional para seu tratamento pedagógico no ensino médio (Flores, 2019), este artigo visa propor uma série de competências semióticas (Tobón, 2005) para facilitar os processos de ensino e aprendizagem da multimodalidade na sala de aula. Estas competências foram formuladas a partir do enfoque sociocognitivo (Tobón, 2005), dos níveis de criação de significados em textos multimodais (Kress e Van Leeuwen, 2001; Kress, 2010) e a taxonomia de Bloom para habilidades cognitivas (Talbert, 2019). Como resultado, eles são propostos como processos complexos, para a aproximação, caracterização, análise, explicação, avaliação e produção de textos multimodais, em interações e contextos socioculturais específicos. Com base na descrição destas competências, o artigo argumenta a necessidade de incorporar uma dimensão semiótica dentro da competência comunicativa no currículo de espanhol (MEP, 2017) e fornece um apoio teórico-metodológico aos docentes para orientar a transposição dos objetivos do currículo nacional, relacionados aos textos multimodais, para a sala de aula. Em conclusão, sugere ligações entre esta proposta e estudos sobre a compreensão de leitura de textos multimodais, a fim de atualizar os processos didáticos e curriculares nacionais, de acordo com o desenvolvimento das múltiplas alfabetizações em nível internacional (Anstey e Bull 2006; Cope e Kalantzis, 2009b; Blanchard e Farstrup 2011; Cooper et al., 2013; Reid and Comber 2004; Walsh 2010).

Palavras-chave: competências, multimodalidade, ensino multimodal, múltiplas alfabetizações, semiologia. 


\section{INTRODUCCIÓN}

El plan de estudios costarricense para la asignatura de español se ha fundamentado sobre el desarrollo de la competencia comunicativa (Lomas, 1999; citado en MEP, 2017), la cual se considera crucial para el acceso general de las asignaturas curriculares, y para el ejercicio de una ciudadanía crítica y responsable. Esta competencia es abordada mediante objetivos de interacción oral y escrita, la lectura crítica de textos de diversa tipología, así como el desarrollo de destrezas para la producción de textos orales y escritos de múltiples géneros.

La competencia comunicativa, como marco general, se explica integrada por las siguientes subcompetencias: lingüística, sociolingüística y sociocultural, discursiva o textual, estratégica, semiológica y lectora o literaria (Lomas, 1999; citado en MEP, 2017, p. 8). Si bien todas ellas constituyen aristas para abordar el fenómeno comunicativo, destaca la ausencia de una competencia semiótica, es decir, de una línea de comunicación enfocada en las destrezas para el manejo de los modos de representación -lingüísticos, visuales, auditivos, gestuales, táctiles espaciales- que se articulan en las prácticas discursivas contemporáneas (Kress, 2003, 2005; Bezemer, y Kress, 2008; Jewitt, 2008; Cope y Kalantzis, 2009a, 2009b).

En su ausencia, la más cercana podría considerarse la subcompetencia semiológica, por cuanto trasciende el sistema lingüístico y concierne la "habilidad para interpretar los elementos iconoverbales empleados por la publicidad y los diversos medios de comunicación" (MEP, 2017, p. 8). Sin embargo, pese a su relevancia, esta última no puede dar cuenta, por sí sola, de las transformaciones semióticas en las prácticas sociales (Kress, 2010), del carácter inherentemente multimodal de las formas de representación contemporáneas (Cope y Kalantzis, 2009a) y del impacto de esta dimensión semiótica en la alfabetización (Kress et al., 2001; Albers y Harste, 2007; Cope y Kalantzis, 2009a).

Pese a no incorporar una competencia semiótica, el plan de estudios sí incluye, dentro de la lista de textos por abordar, géneros con una construcción que los acercaría a la categoría de multimodales, por ejemplo: novela gráfica, tira cómica, grafiti, afiche con anuncio publicitario, video de anuncio publicitario y cine (MEP, 2017, p.193). Ahora bien, estos no aparecen definidos como tales ni tampoco se explicitan los fundamentos de su naturaleza semiótica, lo cual impide al profesorado construir pautas didácticas claras y sistemáticas para afrontarlos, con propiedad, en el salón de clases.

Para el análisis de todos los géneros anteriores, el plan de estudios recomienda la propuesta de Ramírez y Solano (2016), la cual se utiliza en función de textos tanto literarios como no literarios (MEP, 2017, p 16 - 19). La propuesta comprende cuatro fases de lectura interconectadas: natural, de ubicación, analítica, interpretativa y explicativa. Además, es muy valiosa por su amplio respaldo teórico, su solidez y su ejemplificación práctica, que dotan al profesorado de lineamientos claros y explicativos. Su mayor desventaja radica en que, si bien considera la naturaleza semiótica de los textos literarios (Ramírez y Solano, 2016, pp. 19 - 27), no contempla su carácter multimodal.

Además, pese a que el plan de estudios establece una distinción curricular entre y, y construye los objetivos de aprendizaje en torno a esta; no define con claridad la diferenciación. Los conceptos, particularidades de cada tipo de textos, criterios o autores de referencia para su categorización no están explícitos, lo cual genera ambigüedad interpretativa. En estas circunstancias, es aún más evidente que se incentiva al profesorado a trabajar con tipologías de textos y objetivos de análisis multimodal, sin el suficiente apoyo didáctico, teórico o metodológico para un abordaje en toda su especificidad.

En el ámbito internacional, Cope y Kalantzis (2015) elaboran una propuesta pedagógica desde la perspectiva de las alfabetizaciones múltiples, cuya finalidad es, precisamente, ofrecer este marco pedagógico para la aproximación didáctica a los textos multimodales. Su modelo es muy valioso para formular esta propuesta, por cuanto recurre a cuatro tradiciones pedagógicas distintas para explicar la alfabetización múltiple. Como resultado, la define desde una óptica semiótica, pero a la vez educativa y curricular, que 
posibilita trabajar la alfabetización según cuatro aristas interconectadas: la experiencia, la conceptualización, el análisis y la aplicación.

En contextos escolares, la noción multimodalidad de se enfoca en visibilizar la multiplicidad de recursos semióticos a través de los cuales toman lugar las interacciones de pedagógicas, así como la incidencia de estos modos en los procesos de enseñanza y aprendizaje (Cope y Kalantzis, 2009a). Se debe recordar que ni la naturaleza de los contenidos curriculares es únicamente lingüística, ni la mediación y el aprendizaje toman lugar, en exclusiva, a través de este recurso. La representaciónde los discursos escolares es un fenómeno inherentemente multimodal (Kress et al., 2014), así como la mediación y cognición (Gibbons, 2012). En concordancia, la multimodalidad advierte sobre la incidencia de los recursos de creación de significados que se configuran en ambos fenómenos.

Por otra parte, la multimodalidad en contextos educativos debe entenderse en el marco de los cambios en la producción del conocimiento (Kress, 2010), y la profusión de prácticas sociales y textos multimodales, con los cuales entra en contacto el estudiantado y que son incluidos en las planificaciones curriculares (Cope, y Kalantzis, 2009a). Estos textos se caracterizan por modelar de forma activa y dinámica los discursos, mediante la articulación de significados en los modos lingüístico, visual, auditivo, gestual y espacial, entre otros. En coherencia, tanto el profesorado como el estudiantado, ameritan un cuerpo de competencias semióticas, que los preparen para su comprensión y producción, tanto dentro como fuera del aula.

En respuesta a los objetivos de aprendizaje relacionados con textos multimodales, en el plan de estudios de español costarricense (MEP, 2017), y la escasez de propuestas didácticas a nivel nacional para orientar el tratamiento pedagógico de la multimodalidad en clases de secundaria (Flores, 2019), el objetivo del trabajo es proporcionar un marco teórico y metodológico de referencia, a fin de guiar al profesorado en la trasposición de los objetivos del currículo nacional, relacionados con textos multimodales, y su implementación concreta en el aula. Para ello, se toma como referencia el modelo anterior (Cope y Kalantzis, 2015) el cual amplía y reelabora en función del contexto costarricense y los intereses del plan de estudios de la asignatura de español del MEP (2017).

La propuesta nace como un insumo para coadyuvar a la mediación pedagógica del profesorado y presenta una serie organizada de competencias (Tobón, 2005) de naturaleza semiótica para facilitar los procesos de enseñanza y aprendizaje de la multimodalidad en el aula. En su función mediadora, también pretende ser un recurso altamente operativo para el profesorado, es decir, modelar una ruta concreta para la interacción con textos multimodales, la planificación de las estrategias didácticas, la construcción de materiales didácticos y el diseño de situaciones de aprendizaje con textos de esta naturaleza.

Las competencias semióticas tienen como propósito orientar la aproximación, la caracterización, el análisis, la explicación, la evaluación y la producción de textos multimodales. Desde el enfoque sociocognitivo (Tobón, 2005), estas se definen como procesos complejos que requieren la acción-actuación-creación semiótica del individuo en entornos socioculturales específicos. Así, no se conciben como un conjunto de comportamientos externos observables; sino como estructuras cognitivas y afectivas, construidas sobre la articulación de conocimientos, procedimientos y actitudes, que permiten al sujeto comprender y producir textos multimodales en sus interacciones sociales.

Las competencias de esta propuesta se plantean de forma que aborden los niveles de creación de significados en textos multimodales: discurso, diseño, género, producción y distribución, así como las distinciones entre modos y medios (Kress y Van Leeuwen, 2001; Kress, 2010). Los elementos de la competencia formulados, en su descripción, pretenden abarcar las características semióticas a lo interno de los niveles, así como profundizar en las relaciones entre ellos. Como resultado, se persigue un abordaje integral de los textos multimodales en tanto artefactos semióticos complejos y multidimensionales (Bateman, 2008; Kress, 2010).

Por otra parte, la organización de las competencias está pensada desde la taxonomía de Bloom para las habilidades cognitivas, razón por la cual se plantean desde las tareas más simples hasta las más complejas 
(Talbert, 2019). A su vez, a lo interno de las competencias, los elementos van escalonados de lo más simple a lo más complejo. Esta distribución pretende dotar de un carácter procesual al trabajo con los textos multimodales, articular de forma lógica las competencias necesarias para su análisis y procurar un desarrollo entrelazado -y sucesivo- de los elementos indicados a lo interno de cada una de ellas.

Para la adecuada comprensión de las competencias, cabe precisar cuatro constructos teóricos relevantes, que aparecen de forma recurrente y transversal en esta propuesta. En primer lugar, el discurso se comprende como los conocimientos socialmente construidos sobre la realidad o un aspecto de esta, según los intereses de los actores de los contextos (Kress y van Leeuwen, 2001). La aclaración persigue diferenciar el constructo del discurso como (producción de un escrito). En el sentido aquí empleado, el discurso corresponde al material ideológico de origen sociocultural, que se realiza a través de los distintos modos, en los textos multimodales, y que requiere una valoración crítica (Van Dijk, 1996; Kress, 2010; Santander, 2011).

Por otra parte, en el presente trabajo, los modos se comprenden como sistemas organizados de recursos semióticos, disponibles socioculturalmente para la creación de significados y que permiten satisfacer las necesidades representacionales y comunicativas de los creadores de signos (Kress, 2010; Kress y van Leeuwen, 2001; van Leeuwen, 2005). Por su parte, los medios se conciben como la sustancia física, orgánica, modelada socioculturalmente como superficie para instanciar los signos. Así, corresponden a la dimensión material en la cual se realiza de manera concreta y difunde el modo (Kress y van Leeuwen, 2001; Bezemer y Kress, 2008).

Debido a su naturaleza, los constructos de modos y medios no se presentan en una competencia propia, separada de las demás, sino que se integran en forma de elementos específicos a lo interno de todas las competencias. Ya que el potencial de significado de modos y medios depende de su uso contextualizado, en función de ciertos discursos, géneros e interacciones; se considera poco recomendable estudiarlo desligado de estos últimos, pues se incurriría en una incoherencia teórica (Kress y van Leeuwen, 2001; Kress, 2010).

Por su parte, como texto multimodal se considera cualquier "fenómeno sociocultural, cuyos significados discursivos se realizan mediante la articulación de dos o más modos semióticos" (Kress y van Leeuwen, 2001, p. 40, traducción propia). La definición permite incluir tanto las articulaciones de los discursos que poseen la forma más convencional -v.g. un cuento, un anuncio publicitario)-, como aquellas interacciones con una apariencia menos típica -v.g. la música, los programas televisivos-, (Kress y van Leeuwen, 2001; van Leeuwen, 2005).

Según se observa, este constructo no restringe el "texto" al ámbito estrictamente discursivo, sino que lo concibe como -a la vez-como un producto y un proceso social, en respuesta a las necesidades e intereses de los creadores de signos (Kress, 2010; van Leeuwen, 2005). La versatilidad en la definición dota de una amplia riqueza teórica y metodológica a esta propuesta, ya que permite emplearla en coherencia con el programa del MEP (2017) y los tipos de textos que se proponen y, al mismo tiempo, para el eventual análisis de un espectro mucho más amplio de prácticas socioculturales.

Además, esta definición se sitúa por encima de la distinción entre textos literarios y no literarios -por lo demás ambigua, como se explicó antes-. La naturaleza multimodal no se relaciona con el carácter literario o no literario del texto, sino con los recursos de significado que lo articulan. En otras palabras, tanto textos literarios como no literarios (MEP, 2017) pueden ser multimodales, ya que este último rasgo depende de los modos articulados, no de la naturaleza del discurso. La salvedad permite emplear el constructo para ambos tipos de textos, independientemente de su posible interpretación en el plan de estudios.

Por último, las competencias aquí formuladas parten de que el texto multimodal es, además, de naturaleza multimedia, si bien no limitan este último concepto al ámbito tecnológico. Es decir, el carácter multimedia no se asocia estrictamente con la construcción o distribución digital del texto -aunque puede estarlo-, sino con la diversidad de modos que lo articulan, los cuales le confieren el potencial para materializarse y difundirse en medios semióticos igualmente diversos y complejos. Por este motivo, en la redacción de las competencias, solo se hace referencia a los textos como multimodales y no multimedia, pues se considera que este último concepto está englobado en el primero. 


\section{DESARROLLO DEL TEMA}

Una vez introducidas las competencias, las secciones posteriores se emplearán para su descripción. Cada competencia se explica en un apartado específico, según los siguientes tres aspectos. El primero de ellos son los elementos de la competencia, los cuales se definen como desempeños específicos que componen la competencia identificada y cuyo logro supone el desarrollo de las destrezas requeridas (Tobón, 2005). En otros términos, el desarrollo global de la competencia requiere el accionar de los elementos que la componen, los cuales, a su vez, responden a procesos cognitivos, procedimentales y actitudinales concretos.

La enunciación de cada elemento se complementa con una serie de preguntas de orientación. Estas son herramientas a disposición del profesorado para comprender, con mayor profundidad, los procesos implicados en el desempeño de cada elemento. Es decir, para el desarrollo de estos últimos, se recomienda tomar como referencia la pregunta formulada y procurar su resolución, a fin de activar los procesos cognoscitivos asociados. De igual manera, el logro del elemento puede valorarse en relación con las respuestas formuladas para las interrogantes.

El tercer aspecto consiste en una explicitación de los supuestos teóricos que respaldan cada elemento, a fin de robustecer la descripción de la competencia. En primer lugar, se explican las concepciones sobre los textos multimodales y la semiosis, de las cuales se parte para construir los elementos, los cuales justifican su formulación y la redacción de los enunciados tal cual se realiza. Por otra parte, los supuestos aclaran la escogencia de las preguntas orientadoras y se relacionan con la indagación que solicitan. Así, se establecen los límites conceptuales de los fenómenos $y$, por lo tanto, posibles caminos de respuesta para las interrogantes planteadas.

Finalmente, ante la ausencia de una explicación teórica sobre la semiótica social multimodal en el plan de estudios del MEP (2017), estos supuestos pretenden familiarizar al profesorado con este campo y sus relaciones con la lingüística textual. Condensan los fundamentos más relevantes y visibilizan su aplicación, de forma concreta, en función de las competencias, para facilitar la trasposición en clase, especialmente, por parte de aquellas personas que no han tenido contacto previo con la semiótica social multimodal. En ese sentido, las referencias bibliográficas quedan a disposición de aquellos que deseen profundizar en las temáticas.

\section{Competencias semióticas para la alfabetización multimodal}

\section{Competencia 1: Reconocer la naturaleza multimodal y multimedia de los discursos, en textos de diversos géneros textuales.}

La tabla 1 sintetiza los elementos que constituyen esta competencia, así como las preguntas orientadoras sobre los procesos implicados en el desempeño de cada elemento.

TABLA 1

Elementos de la competencia 1 y preguntas orientadoras

Elementos de la competencia

1. Determinar los modos articulados en textos multimodales de diversos géneros textuales.

2. Describir el diseño multimodal de los textos multimodales de diversos géneros textuales.

3. Identificar los soportes (medios) en los que se materializan y distribuyen los textos multimodales de diversos géneros textuales.

\section{Preguntas orientadoras}

¿Qué modos están presentesen elconjunto de textos dados?

¿Cómo está diseñado el texto? Además de los modos que lo constituyen, ¿cuáles son sus partes, cómo se organizan estos en el espacio?

¿A través de cuál materialidad (sustancia física) se realizan los textos?

Fuente: Elaboración propia (2020). 
Esta primera competencia se enfoca en activar la percepción de los textos desde una mirada multimodal. Para ello, sus elementos aproximan sucesivamente al intérprete hacia el reconocimiento de los modos instanciados, su configuración en el diseño textual, así como a generar razonamientos que distingan la noción de modos y medios.

El primer elemento parte del supuesto de que los discursos son susceptibles de representarse y comunicarse a través de distintos modos, los cuales pueden distinguirse según los siguientes sistemas: auditivos, orales, escritos, visuales, espaciales, táctiles, gestuales y auditivos (Cope y Kalantzis, 2009b). Sistemas que incluyen tanto la lengua verbal como escrita; los gestos, las postura, los movimientos del cuerpo o sus miembros y los desplazamientos en el espacio; el uso de recursos sonoros y visuales: imágenes (tanto fijas como en movimiento), colores, figuras, íconos, tablas, cuadros, gráficos, fotografías, dibujos, mapas y presentaciones visuales (Lemke, 1998; Cope y Kalantzis, 2009b).

El segundo elemento enfrenta al intérprete con la composición del texto multimodal. Para ello, se sugiere, en primer lugar, llevar a cabo un reconocimiento visual de las unidades mínimas que los constituyen y, posteriormente, una identificación de agrupaciones formadas por esta últimas (Bateman, 2008). La aproximación permite desmenuzar la composición mínima del texto según criterios perceptuales -no funcionales- $y$, por tanto, en ulteriores etapas de análisis, posibilita explicitar con mayor claridad el impacto en la significación desde un nivel básico, esquemático. Para llevar a cabo el proceso de forma más didáctica, se sugiere al profesorado utilizar el Modelo de Análisis de Géneros Multimodales (MAGEM)1.

Finalmente, el tercer elemento parte del supuesto de que los discursos, modos y medios constituyen dimensiones interconectadas de los textos multimodales (Kress y Van Leeuwen, 2001; Kress, 2010). Los discursos se realizan a través de modos, los cuales se materializan a través de medios. Cada modo, por lo tanto, se vincula a una materialidad, en la cual toma lugar e incide en los significados discursivos. Como se mencionó anteriormente, el reconocimiento de la multimodalidad implica, también, la identificación de su carácter multimedia.

\section{Competencia 2: Caracterizar la construcción semiótica de los géneros textuales, en textos multimodales.}

La tabla 2 sintetiza los elementos que constituyen la competencia, así como las preguntas orientadoras sobre los procesos implicados en el desempeño de cada elemento.

TABLA 2

Elementos de la competencia 2 y preguntas orientadoras

Elementos de la competencia

1. Asociar diseños semióticos de textos multimodales, con géneros textuales específicos.

2. Diferenciar la representación de los discursos que realizan los géneros, según el diseño semiótico.

3. Contrastar los diseños semióticos de los géneros textuales, según distintos medios de producción y distribución de los textos multimodales.

\section{Preguntas orientadoras}

¿Mediante cuáles modos y diseños semióticos se realiza, típicamente, el género textual (noticia, anuncio publicitario, etc.)?

¿Diseñar de forma distinta un mismo género (por ejemplo, noticia) modifica la manera en que interpretamos su contenido (su discurso)? ¿El uso o desuso de ciertos modos incide en la interpretación del contenido discursivo? Si ya los géneros en sí mismos construyen significados, ¿hay otros significados que suman o restan los modos semióticos utilizados en el género?

¿Los géneros se construyen semióticamente de la misma manera o de formas distintas, según la superficie en la que se materializan y distribuyen (papel, audio, formato digital, video, etc.)? ¿Estas diferencias en la materialidad inciden en el significado? ¿Las personas se encuentran estos textos en las mismas situaciones?

Fuente: Elaboración propia (2020). 
La competencia se enfoca en los géneros textuales, tiene como fin abordar su diseño semiótico, así como la incidencia de este último en el discurso. Así, pretende introducir las relaciones entre géneros, discursos, modos y medios. El género se concibe como un filtro, que incide en los diversos niveles de estructuración del texto: "el nivel de las restricciones, el nivel de la organización discursiva, determinada por las instrucciones situacionales y el nivel de las marcas formales, más o menos recurrentes, necesarias para configurar lingüísticamente la organización discursiva" (Charaudeau, 2004, p.1).

El primer elemento parte de que los géneros textuales (noticias, anuncios, novela gráfica, grafiti, entre otros) tienden a realizarse, semióticamente, de la misma manera; es decir, a través de diseños semióticos típicos. Esta regularidad supone la existencia de un patrón de diseño multimodal, particular y recurrente, asociado a los géneros y a su construcción discursiva (Kress et al., 2001). Como resultado, estos se experimentan como prácticas sociales reconocibles, constituidos por configuraciones relativamente regulares y estables (Van Leeuwen, 2005; Kress, 2010).

El segundo elemento sostiene que los modos (oralidad, escritura, color, entre otros) pueden repetirse a través de los géneros textuales (noticia, anuncio publicitario, entre otros); no obstante, crean significados distintos según varíe el diseño del texto (Kress, 2005; 2010). Por este motivo, se considera relevante comparar un mismo género textual con usos modales distintos, ya que permite valorar los significados que los modos "agregan" o "sustraen" al género en sus diversas manifestaciones concretas; es decir, sus implicaciones semióticas y discursivas.

El último elemento destaca que los medios de materialización de los textos también inciden en el potencial de los modos y en los significados finalmente representados (Kress y Van Leeuwen, 2001; Kress, 2005, 2010). Por tanto, compara un mismo género textual en soportes distintos, a fin de valorar los significados que el medio agrega al género e, incluso, a los modos semióticos. Además, posibilita explorar las diferencias en los procesos de consumo, lectura e interpretación textual que implica la selección de los diversos medios.

Las siguientes dos competencias (3) y (4) parten de la relevancia de las situaciones de enunciación y de comunicación en las que se ubica el texto. La primera de ellas comprende el contexto situacional en que se formula el texto -se enuncia-, por lo que está relacionada a su producción, circulación y recepción. Atender a la enunciación implica valorar el estatus y relaciones de poder subyacentes entre las instancias de producción y recepción, así como los mecanismos de control que subyacen a la distribución textual. Si bien la descripción parece sugerir que las tres instancias se relacionan de forma unidireccional, la propuesta sostiene que las tres actúan como actores en la enunciación, y que las tres dimensiones son capaces de influenciarse entre sí.

La situación de comunicación se refiere al contexto situacional en que ocurre el acto comunicativo como tal, por cuanto no existe acto de lenguaje fuera del contexto (Charaudeau, 2006) y porque, tanto la significación como el empleo de los modos semióticos, responden a los intereses de los creadores de signos en situaciones comunicativas y comunidades socioculturales específicas (Van Leeuwen, 2005; Kress, 2010). Una situación de comunicación se define como aquella en donde se encuentran "los actores (dos al menos) que comunican, es decir que intercambian propósitos con el interés de lograr una cierta inter-comprensión, y cuyo sentido depende, por una parte, de las condiciones en las cuales se realiza el intercambio" (Charaudeau, 2006, p. 39).

Cada situación comunicativa está modelada según, al menos, cuatro componentes (Charaudeau, 2006, p. 40):

1. Su finalidad ¿para decir qué?;

2. la identidad de los participantes (¿quién comunica con quién?);

3. el tema que constituye el objeto del intercambio entre los dos interlocutores (¿a propósito de qué?);

4. las condiciones físicas y materiales en las que se desarrolla la comunicación (¿en qué circunstancias se comunica?). 
Los elementos de estas competencias, por consiguiente, buscan caracterizar las interacciones comunicativas, así como explicar los procesos de significación de los textos multimodales, en relación con estas constantes.

Además de los cuatro aspectos anteriores, cabe destacar que toda interacción comunicativa se rige a partir de un contrato comunicacional (Charaudeau, 2006), razón por la cual el constructo se incluye en la competencia. El contrato de comunicación opera como un acuerdo, implícito o explícito, entre los participantes. Estructura el intercambio y define ciertas condiciones o parámetros para su realización y los actos que allí se producen, de modo que sean reconocidos como válidos (Charaudeau, 2006). La aceptación del contrato alinea las intencionalidades del sujeto comunicante y el sujeto receptor-interpretante, así como su reciprocidad.

\section{Competencia 3: Caracterizar la situación de enunciación de los textos multimodales.}

La tabla 3 sintetiza los elementos que constituyen la competencia, así como las preguntas orientadoras sobre los procesos implicados en el desempeño de cada elemento.

TABLA 3

Elementos de la competencia 3 y preguntas orientadoras

\section{Elementos de la competencia}

1. Definir las instancias de producción de textos multimodales.

2. Caracterizar los procesos de circulación de textos multimodales en relación con los medios semióticos.

3. Caracterizar el momento de lectura de los textos multimodales según su circulación y los medios semióticos.

4. Caracterizar la audiencia en textos multimodales.

5. Establecer la relación entre las instancias de producción y audiencia de textos multimodales.

6. Determinar el tipo y grado de interacción comunicativa en la que se produce, circula y se recibe el texto multimodal.

7. Caracterizar el contrato comunicacional entre instancia de producción y audiencia, que está implicado en esa interacción comunicativa (género).

\section{Preguntas orientadoras}

¿Quién produce el texto? ¿Cuáles son las características de esa instancia? ¿Cuál es su rol en la sociedad? ¿Es un actor con/sin poder, experto/no experto, confiable/ no confiable? ¿Puede tener intereses específicos esta instancia?

¿A través de cuáles canales se distribuye el texto en este contexto sociocultural? ¿Circulan libremente por cualquier canal? ¿Se paga o es gratis? ¿Cualquier persona tiene acceso a esos canales de distribución?

¿Bajo qué circunstancias se lee el texto? ¿De cuál manera inciden estas circunstancias de lectura en la recepción del texto? Por ejemplo: periódico con calma, noticias durante el desayuno, navegar en el teléfono, en el bus, podcast, entre otros.

¿A quién va dirigido el texto? ¿Cuáles son sus características? ¿Quién lee el texto? ¿Cuáles son sus características? ¿Tienen las mismas características el destinatario y el lector real?

¿Cuál nivel de distanciamiento social existe entre la instancia de producción y la audiencia del texto? ¿Existe una relación jerárquica, solidaria, de cercanía o distancia social? ¿De cuál manera incide esta relación entre la instancia de producción y la audiencia, en la lectura y la recepción del texto?

Las características de la circulación del texto, ¿qué tanta interacción permiten establecer entre las instancias de producción y la audiencia? ¿La interacción es sincrónica u ocurre posterior a la producción del texto? ¿Cómo incide este nivel de interacción en la lectura del texto que realiza la audiencia?

¿Cuáles expectativas comunicativas posee la audiencia sobre el texto, según el género? ¿Cuáles expectativas comunicativas posee la instancia de producción para el texto, según el género? ¿Cuál es la naturaleza de estas expectativas, tanto por parte de la instancia de producción como de la audiencia? ¿Cómo inciden estas expectativas en la lectura? ¿Cómo inciden en la circulación y producción del texto?

Fuente: Elaboración propia (2020).

Esta competencia está enfocada, específicamente, en describir las condiciones contextuales y situacionales que rodean la producción, circulación y recepción del texto. El primer elemento parte de las instancias de producción, las cuales considera como actores sociales con estatus, legitimidad, capital simbólico y que participan de relaciones de poder (Charaudeau, 2006). Así, sostiene que las características también inciden en las posibles finalidades al producir cada texto en particular. 
El segundo elemento aborda los canales de circulación, los cuales considera como determinantes para el acceso a los textos, la rapidez de su divulgación y alcance. Estos canales inciden, a su vez, en los momentos de lectura y, con ello, en los procesos de interpretación (Charaudeau, 2006). Según estos supuestos, el elemento pretende rastrear diferencias interpretativas entre la lectura, por ejemplo, de un periódico digital, un posteo en redes sociales, un noticiero en televisión, una revista impresa.

El tercer elemento parte de que los individuos se enfrentan a los textos dentro de múltiples interacciones, es decir, leen los textos en distintos momentos, lo cual se asocia con el grado de atención que les pueden prestar e, incluso, su procesamiento cognitivo. Así, el elemento sostiene que, además de los canales de circulación, las condiciones particulares de los momentos de lectura pueden incidir en los procesos de interpretación textual (Charaudeau, 2006).

El cuarto elemento considera el papel de audiencia en el análisis situacional, el cual está determinado por el texto y por la circulación. Puede haber diferencias entre el destinatario (quién se pensó para que leyera el texto) y la audiencia (quien finalmente lee el texto). Asimismo, los efectos que puede tener un texto dependen de a quién está dirigido y quién lo recibe (Livingstone y Das, 2013). Por estas razones, es importante valorar, con base en las características del texto y su circulación, si destinatario y lector son los mismos o si, por el contrario, son entidades diferentes.

En el quinto elemento, el análisis situacional considera los tipos de relaciones entre las instancias de producción y audiencia, las cuales inciden en el acceso e interpretación del texto. Estas últimas pueden estar preestablecidas y conocidas por ambas partes; por ejemplo, entre medio de comunicación con sus lectores, o la relación profesorado-estudiantado (Livingstone y Das, 2013). En otros casos, dichas relaciones solo se ven evidenciadas por la intención del productor del texto, construida a nivel textual. Las relaciones pueden variar y manifestarse como jerárquicas o solidarias, de cercanía o distanciamiento, entre otras (Charaudeau, 2006).

Para la formulación del sexto elemento, se parte de que los intercambios comunicativos se diferencian según el grado de interacción que posibiliten entre los implicados, es decir, de acuerdo con la alternancia de roles entre sus participantes. Cuando existen cambios de roles, la construcción del sentido es conjunta, mientras que cuando no los hay, tiende a ser un proceso más individual. Además, el grado de interacción puede generar diferencias entre los momentos de producción y circulación, lo cual afecta la recepción del texto. Así, esta última puede ser diferida -en un momento distinto de la producción- o sincrónica -al mismo tiempo de la producción-.

El último elemento aborda el contrato comunicacional entre instancia de producción y audiencia, considerando las expectativas de los interlocutores acerca del intercambio. Estas últimas se encuentran en estrecha relación con, al menos, dos elementos: el género textual y la relación entre los participantes. Como resultado, pueden ser, al menos, de tres tipos, cuya caracterización es relevante a fin de profundizar en la construcción semiótica del texto:

1. Expectativas de interacción (entre instancia de producción y audiencia);

2. expectativas discursivas (tipo de contenidos);

3. expectativas de finalidad (propósito del texto). (Charaudeau, 2004; 2006)

\section{Competencia 4: Explicar los significados en textos multimodales de diversos géneros textuales, a partir de relaciones entre el texto y su contexto, la situación comunicativa, las instancias de producción y recepción, y el ámbito textual mismo.}

La tabla 4 sintetiza los elementos que constituyen la competencia, así como las preguntas orientadoras sobre los procesos implicados en el desempeño de cada elemento. 
TABLA 4

Elementos de la competencia 4 y preguntas orientadoras

\section{Elementos de la competencia}

\section{Dimensión del significado contextual}

1. Caracterizar las entidades y procesos de representación, en relación con el contexto sociocultural de los textos multimodales de diversos géneros textuales.

\section{Dimensión del significado situacional}

2. Caracterizar la situación comunicativa y su inscripción en los textos multimodales de diversos géneros textuales.

\section{Dimensión del significado interpersonal}

3. Inferir las características de la audiencia meta según el diseño semiótico de textos multimodales, de diversos géneros textuales.

4. Definir las relaciones interpersonales entre las instancias de producción y recepción manifestadas por medio de los recursos semióticos y modos de textos multimodales de diversos géneros textuales.

\section{Dimensión del significado textual}

5. Reconocer los modos a través de los cuales se realizan los discursos, en textos multimodales de diversos géneros textuales.

6. Identificar el contenido microtextual representado en cada modo semiótico, en textos de diversos géneros.

7. Determinar el contenido microtextual a partir de las relaciones entre los distintos modos, en textos multimodales de diversos géneros textuales.

8. Establecer el contenido macrotextual elaborado a través de los distintos modos, en textos multimodales de diversos géneros textuales.

9. Contrastar el contenido microtextual de textos multimodales, de acuerdo con los medios de producción y distribución.

\section{Preguntas orientadoras}

¿Qué significados del contexto sociocultural en que se enmarca el texto se evidencian a través de los recursos semióticos y modos? ¿Qué referentes, rasgos, entidades, aspectos, etc., son interpretables, exclusivamente, al atender al contexto sociocultural del texto?

¿En qué situación comunicativa se realiza el texto? ¿Quiénes participan en la interacción comunicativa? ¿Qué referentes de la situación comunicativa se incorporan en el texto? ¿Cuáles de estos referentes son necesarios para la interpretación? ¿Qué rasgos lingüísticos y semióticos de la situación comunicativa caracterizan a los participantes de la interacción?

¿A quién va dirigido el texto, el discurso representado? ¿Qué características se atribuyen a la audiencia?

¿Cuál tipo de relación interpersonal se establece entre las instancias de producción y recepción del texto? ¿Jerárquica, solidaria, cercana, distante? ¿Cuál es el registro del texto? ¿Formal, informal, coloquial? ¿Cuál es la intención comunicativa de la instancia de producción sobre la audiencia, a partir de los significados de texto multimodal? Es decir, ¿cuáles actos de habla están presentes en el texto?

¿Cuáles discursos se representan en el texto? ¿Qué modos semióticos representan los discursos instanciados?

¿Qué contenido proposicional es representado a través de cada uno de los modos?

¿Existen proposiciones construidas a partir de la relación entre dos o más modos? ¿Cuál aspecto de la proposición es comunicada por cada modo? ¿Cómo se relacionan semánticamente las proposiciones de los modos entre sí?

¿Cuál es el contenido semántico global que aborda el texto? ¿Cuál es, en términos generales, su tema? ¿Cómo se relacionan, en un plano más general, las proposiciones para construir esta temática?

¿Existen diferencias en la representación del contenido microtextual según el medio en que se materializa y distribuye? ¿Cuál significados agrega/restan los medios al discurso?

Fuente: Elaboración propia (2020).

La competencia se enfoca en los procesos de significación. Sus elementos buscan explicar los significados a partir de la interacción del texto con su contexto sociocultural, situacional, las relaciones entre las instancias de producción y recepción, y la construcción textual misma. En concordancia, los elementos se han organizado en las siguientes dimensiones: sociocultural, comunicativa, interpersonal y textual. Cabe aclarar que la división responde intenciones meramente didácticas, ya que se consideran aristas interconectadas de la semiosis.

La primera dimensión se concentra en las relaciones entre el texto y el entorno sociocultural. El primer elemento supone que existen significados asociados a los modos semióticos, los cuales evidencian que el texto se inserta en un contexto social determinado. Estos incluyen referentes, entidades, aspectos sociales y culturales, cuya interpretación se haya estrechamente enlazada con el contexto social. Así, si la audiencia no remite a un marco sociocultural para identificar los referentes, la interpretación del texto se ve comprometida (Van Dijk, 1993). 
La segunda dimensión retoma los significados que emergen de las relaciones entre el texto y la situación comunicativa, las cuales fueron abordadas con mayor detalle en la competencia anterior (3). El segundo elemento sostiene que las características del entorno comunicativo se ven representadas en los textos, tanto en la selección de recursos semióticos específicos como en referencias concretas al momento, la locación, los participantes o la misma interacción comunicativa (Charaudeau, 2004, 2006; Kress, 2010). Por tanto, integra los significados socioculturales con los situacionales.

La tercera dimensión de análisis del significado corresponde a los vínculos interpersonales entre las instancias de producción y recepción del texto. El tercer elemento parte de que la construcción textual responde a un interés motivado; por lo que no es gratuita, sino contextualizada y con propósitos comunicativos particulares (Kress, 2010). La configuración semiótica y discursiva del texto está alineada con los propósitos y, por tanto, puede arrojar pistas sobre la instancia de producción, el destinatario y visibilizar diferencias respecto de su audiencia real (Kress at al., 2001). Además, las relaciones entre la instancia de producción y recepción se evidencian en los modos articulados en los textos, por lo que es pertinente determinar su naturaleza.

El cuarto elemento procura interpretar la finalidad de la instancia de producción, sobre la de recepción, con ese texto, para lo cual hace referencia a las intenciones comunicativas representadas en el texto, en forma de actos de habla implícitos o explícitos: persuadir, llevar a la acción, presentar conocimiento nuevo, comprometerse con actos futuros, entre otros (Charaudeau, 2006). Por lo demás, también sostiene que la escogencia del registro (formal o informal) supone una relación interpersonal específica y puede vincularse con las intenciones comunicativas y los rasgos de las instancias de producción y recepción (Charaudeau, 2006).

La última dimensión corresponde al nivel textual y toma como objeto de análisis el texto mismo. El quinto elemento solicita un repaso sobre los modos empleados en cada texto, por cuanto su presencia incide en los significados analizados en los elementos subsecuentes de la competencia (Kress y Van Leeuwen, 2001; Van Leeuwen, 2005; Kress 2010). En el sexto elemento, el reconocimiento semiótico inicial se traslada hacia el análisis La microestructura se considera como cada una de las proposiciones de significado construidas por los modos de forma individual, las cuales, vinculadas semánticamente entre sí, dan soporte a la idea general de un texto (Van Dijk, 1980a, 1980b).

En el sexto elemento, la descripción microtextual se enfoca en analizar el significado a lo interno de cada uno de los modos, para la cual es necesario recurrir a distintas dimensiones del análisis visual, además del verbal:

1. Descripción de lo que se ve,

2. interpretación de lo que se ve (la cual puede ser referencial o metafórica) y

3. la interpretación en relación con el contexto histórico y social.

En el sétimo elemento, el significado microtextual considera las relaciones entre modos, la articulación de sus proposiciones. Aquí, se debe considerar que, a nivel microtextual, también se despliegan relaciones lógico-semánticas entre los segmentos textuales y modalidades que organizan y estructuran el contenido global del texto. Para el análisis de las últimas se ofrecerán referencias bibliográficas más adelante.

El octavo elemento conduce el análisis del significado al nivel macrotextual. La macroestructura se relaciona con el contenido semántico global, en otras palabras, con el asunto o tema abordado por el texto. El sentido global es el que permite concebirlo como una unidad de comunicación, con un núcleo informativo central, de ahí su relevancia (Van Dijk, 1980a, 1980b). Al vincular los aspectos previos, se observa que el sexto elemento (6) aborda el contenido semántico de cada modo aislado (en la medida de lo posible); el séptimo (7) determina las relaciones entre modos y sus distintas proposiciones textuales; el octavo (8) estudia su articulación global en el texto, como unidad de sentido. 
En el noveno elemento, el análisis de la microestructura se conecta con los medios semióticos, es decir, requiere profundizar en las relaciones entre los soportes y los significados textuales. Se parte de que las superficies de materialización y distribución de los textos inciden en el potencial de los modos y, finalmente, en los significados discursivos representados (Kress y Van Leeuwen, 2001; Van Leeuwen, 2005; Kress 2010). Es decir, para el análisis multimodal, el medio es también significativo e incide en la semiosis.

\section{Competencia 5: Explicar los cambios de significado por procesos de traducción semiótica, en textos multimodales de diversos géneros textuales}

La tabla 5 sintetiza los elementos que constituyen la competencia, así como las preguntas orientadoras sobre los procesos implicados en el desempeño de cada elemento.

TABLA 5

Elementos de la competencia 5 y preguntas orientadoras

Elementos de la competencia

1. Determinar las relaciones lógico semánticas entre modos semióticos, en los textos multimodales de diversos géneros textuales.

2. Identificar procesos de transformación y transducción semiótica, en textos multimodales de diversos géneros textuales.

3. Diferenciar los alcances de representación de los modos, según los procesos de transformación y transducción semiótica, en textos de diversos géneros textuales.

4. Explicar los cambios modales y discursivos ocurridos en los géneros textuales, como parte de los procesos de transformación y transducción.

5. Determinar modificaciones en los medios de producción y distribución, como parte de los procesos de transformación y transducción.
Preguntas orientadoras

¿Cómo se relacionan los recursos visuales entre sí? ¿Cómo se relacionan los recursos verbales con los visuales?

¿Existen significados "similares" representados en más de una modalidad? ¿Varios aspectos de un mismo discurso son comunicados por diversos modos? ¿El mismo discurso se desplaza de un modo a otro? ¿El mismo discurso se desplaza de un género a otro?

¿Cuáles diferencias existen en el discurso, al emplear distintos modos? ¿Cuáles nuevos significados discursivos (alcances) permiten representar los modos a los que se traducen los significados? ¿Cuáles aspectos discursivos no pueden representar (limitaciones) estos modos, a los que se traducen los significados?

Al desplazar el discurso de un género a otro, ¿existen cambios en los modos empleados? Al desplazar el discurso de un género a otro, ¿existen modificaciones en el discurso mismo?

Al desplazar el discurso de un género a otro, ¿existen variaciones en los medios empleados para la materialización y distribución del texto? ¿Reelaborar los discursos entre diversos modos requiere, a su vez, modificar los medios?

Fuente: Elaboración propia (2020).

En apoyo a la anterior, la competencia profundiza en los procesos de significación intersemiótica. La producción semiótica no se concibe como un proceso unívoco ni unidireccional, sino que exige pasos de significados de un modo a otro, a fin de articular aspectos discusivos a través de múltiples géneros textuales (Kress, 2010). En concordancia, los elementos de la competencia profundizan en las implicaciones de estos movimientos modales, discursivos y genéricos.

En el primer elemento, para el análisis de las relaciones lógico-semánticas, se sigue la clasificación del modelo MAGEM, la cual, a su vez, constituye una reelaboración de las categorías propuestas de Martinec y Salway (2005) y Unsworth (2006). Las relaciones comprenden: elaboración (subdividida a su vez en explicación, exposición, ejemplificación y reformulación), adición, causalidad, contraste, condicionalidad, temporalidad (comprende relaciones de ubicación y secuencialidad), espacialidad y -finalmente- proyección (verbal y mental).

Para el segundo elemento, la propuesta sostiene que los productos y eventos semióticos constituyen representaciones creadas a partir de principios de transformación y transducción (Kress, 2010). El primero 
se refiere a cambios en el ordenamiento y configuraciones de los elementos dentro de un modo, los cuales no afectan sus significados. El segundo, se refiere al paso de significados, expresados en un modo, a otro(s) modo(s), lo cual conlleva modificaciones ontológicas importantes en la entidad semiótica (Kress, 2010). Ambos procesos se incluyen bajo el concepto de traducción semiótica, que refiere a los procesos de cambio en las representaciones y sus implicaciones en el significado.

El tercer elemento busca rastrear variaciones en los alcances semióticos de los modos, según ocurran pasos entre discursos y géneros textuales. El análisis de la traducción semiótica supone que los modos ofrecen distintos alcances para la representación discursiva (Van Leeuwen, 2005; Bezemer y Kress, 2008); motivo por el cual el paso de significados entre modalidades no es equivalente. Puesto que cada modo ofrece potenciales únicos, la realización del discurso en esa modalidad es, asimismo, particular (Kress, 2010).

El cuarto elemento desliza el foco desde los modos hacia los géneros, y explica los cambios modales y discursivos, ocurridos los últimos como parte de los procesos de transformación y transducción. El paso de discursos entre géneros textuales implica también modificaciones en el diseño y, como resultado, en la instanciación modal del discurso (Kress et al., 2001). Así, los procesos de transformación y transducción, que ocurren como parte del cambio entre géneros, influyen tanto las modalidades como los discursos que estas realizan (Kress, 2010).

Finalmente, en el quinto elemento, para el análisis de los procesos de traducción, se debe considerar que los modos poseen materialidades más o menos aptas para su realización, así como los géneros orientan la selección de medios específicos. En consecuencia, el paso de significados entre géneros y modos conlleva decisiones sobre las superficies para materializar los textos y distribuirlos. Por tanto, implica analizar la incidencia de las traducciones en la selección de los medios semióticos (Kress, 2010).

\section{Competencia 6: Evaluar textos multimodales en relación con sus propósitos comunicativos, la audiencia y el contexto sociocultural.}

La tabla 6 sintetiza los elementos que constituyen la competencia, así como las preguntas orientadoras sobre los procesos implicados en el desempeño de cada elemento.

TABLA 6

Elementos de la competencia 6 y preguntas orientadoras

Elementos de la competencia

1. Determinar la pertinencia y finalidad social de textos multimodales, en relación con su contexto sociocultural.

2. Determinar la concordancia entre los participantes de la interacción, el propósito comunicativo y los textos multimodales.

3. Valorar la adecuación de los procesos de circulación de textos multimodales, según la audiencia y los propósitos comunicativos.

4. Evaluar la adecuación del género según la audiencia y los propósitos comunicativos de textos multimodales.

5. Determinar la adecuación del discurso en función del género y los propósitos comunicativos de textos multimodales.
Preguntas orientadoras

¿Cuáles posiciones ideológicas se evidencian en el texto? ¿Cuál es la pertinencia del texto, dentro de su entorno comunicativo yo sociocultural? ¿Cuál es su finalidad social?

¿Quién produce el texto? ¿A quién va destinado? ¿Cuál es el propósito comunicativo del texto? ¿El texto producido es coherente con los participantes involucrados? ¿Permite cumplir el propósito comunicativo con la audiencia?

¿Cuál medio se utiliza para materializar el texto? ¿A través de cuáles canales se distribuye el texto? ¿La audiencia está familiarizada con estos procesos de circulación del texto? ¿Estos procesos permiten la recepción adecuada del texto por parte de la audiencia?

¿A quién está destinado el texto? ¿La audiencia está familiarizada con el género? ¿E género es interpretable por la audiencia? Al emplear el género, ¿la audiencia puede interpretar el discurso, en coherencia con el propósito comunicativo del texto?

¿Cuál tipo de discursos están representados a través de este género? ¿La escogencia de este género para dicho discurso permite cumplir el propósito comunicativo del texto? ¿Cuáles implicaciones en la significación discursiva posee la configuración genérica específica? 
6. Evaluar la construcción macrotextual de textos multimodales.

7. Evaluar la adecuación del diseño semiótico al discurso y al género, en textos multimodales.

8. Evaluar la adecuación del diseño semiótico según los procesos de comprensión de textos multimodales.

9. Valorar el empleo de los modos según su propia gramática y alcances de representación, en textos multimodales de diversos géneros textuales.

10. Valorar el uso de los modos según las características de los géneros y los propósitos comunicativos, en diversos textos multimodales.

11. Evaluar el uso de los modos según los propósitos comunicativos y las instancias de producción y recepción, en textos multimodales
¿El texto es coherente semánticamente? ¿Tiene un claro tema central (global)? ¿Cuáles son los subtemas? ¿Todos los subtemas se relacionan con el tema global?

¿El diseño semiótico representa el discurso de forma que el texto cumpla el propósito comunicativo? ¿El diseño se ajusta a las características del género mismo? ¿Es coherente con su superestructura, macroestructura y microestructura?

¿El diseño semiótico (distribución de los componentes) facilita la lectura del texto? ¿El diseño (distribución de los componentes) facilita la comprensión del texto? (facilita encontrar los significados y las relaciones de y entre los modos).

¿El diseño semiótico del texto evidencia un proceso de orquestación de los modos semióticos? Es decir, ¿un uso articulado, no aislado de los modos? ¿El uso de los modos es coherente con sus propias posibilidades de representación (su propia gramática)? ¿Los modos se utilizan para construir significados que están dentro de sus alcances? ¿La gramática de los distintos modos es adecuada?

¿El género escogido requiere utilizar los modos de una manera determinada? ¿Es posible emplear los modos de otra forma? ¿El uso de los modos es coherente con el género, con su superestructura, macroestructura y microestructura? ¿El uso de los modos y los significados se definen a través de ellos, dentro del género, permite alcanzar el propósito comunicativo del texto? ¿Los significados definidos por los distintos modos aportan al tema global?

¿La audiencia domina la gramática de los modos empleados en el diseño semiótico? ¿Es capaz de interpretar los significados representados a través de los modos?

Fuente: Elaboración propia (2020).

La evaluación corresponde a una habilidad de orden superior (Talbert, 2019). Los elementos planteados en la competencia son numerosos, precisamente debido a su complejidad y por cuanto requieren poner en práctica destrezas ejercitadas en competencias previas. En este nivel, los elementos constituyen una serie gradual e interconectada de acciones, cuyo objetivo es modelar juicios valorativos sobre la construcción discursiva, genérica y semiótica de los textos multimodales, en relación con los propósitos comunicativos del texto mismo, su adecuación a la audiencia y al contexto comunicativo y sociocultural.

El primer elemento sugiere valorar la pertinencia y finalidad social de textos multimodales, en relación con su contexto sociocultural. Su pertinencia se justifica por cuanto ningún texto existe en el vacío, sino situado histórica y socioculturalmente, por lo que evidencia las representaciones e ideologías culturales del contexto en que es producido (Van Dijk, 1993). Además, el texto mismo se resignifica mediante su circulación en el contexto y los procesos de lectura, por lo que la situación comunicativa también es relevante para la evaluación de sus finalidades.

El segundo elemento sostiene que la producción textual implica el desarrollo de destrezas para identificar los rasgos relevantes de las interacciones comunicativas, seleccionar e interpretar las estructuras genéricas más adecuadas respecto de cada situación, los papeles y las relaciones interpersonales. También, supone desarrollar habilidades para la organización textual y la regulación del discurso, con base en el propósito comunicativo del texto dentro de cada interacción, así como las normas sociales puestas en juego (Sánchez, Morales, del Pozo y Díaz, 2006).

El tercer elemento aborda la dimensión de la distribución (Kress, 2010) y valora los procesos de circulación textual en función de los medios semióticos. Parte de la existencia de relaciones estrechas entre los medios y los canales de distribución de los textos, cuya adecuación incide en el cumplimiento último de sus propósitos comunicativos (Charaudeau, 2006). Es decir, los medios modelan, en gran medida, los procesos de circulación que influyen en el acceso al texto por parte de la audiencia así como la realización de las intenciones comunicativas en el contexto sociocultural y situacional. 
El cuarto elemento extiende las implicaciones de los aspectos anteriores. La producción textual supone emplear procedimientos intratextuales y pragmáticos, a fin de modelar el discurso escrito según el contexto situacional y sociocultural (Van Dijk, 1980a, 1980b; Sánchez, Morales, del Pozo y Díaz, 2006). Así, se requiere al creador de signos configurar sus mensajes tomando en consideración la competencia sociolingüística, pragmática y discursiva (MEP, 2017), para garantizar la adecuación del género textual a la audiencia y a los propósitos comunicativos.

El quinto elemento valora la adecuación del discurso en función del género y los propósitos comunicativos. Se sustenta en la medida en que el discurso no existe como realidad abstracta alejada del género textual, el cual lo crea en función de interacciones comunicativas específicas. Así, el género atribuye al texto una configuración representacional, interpersonal y textual particular a través del diseño semiótico (Kress et al., 2001; Kress y Van Leeuwen, 2001). Como resultado, el género incide en los significados discursivos.

Para el sexto elemento, es necesario considerar que, a nivel macroestructural, un texto se considera coherente sólo si lo es también en un plano más global. La coherencia global se evidencia en la construcción del tema o asunto al texto. Al ser posible reconstruir una macroestructura que dé cuenta de las relaciones entre sus partes, puede decirse que el texto es coherente globalmente (Sánchez, Morales, del Pozo y Díaz, 2006).

El sétimo elemento evalúa el diseño semiótico, definidos como el proceso de elaboración de los signos en forma de textos, lo cual implica conectar coherentemente los modos, medios, marcos y sitios de representación, con los intereses del creador de signos y su audiencia meta, dentro de un contexto sociocultural y comunicativo específico (Bezemer y Kress, 2008; Kress, 2010). La valoración del diseño implica relacionar los alcances de representación de cada modo, los intereses del creador y la complejidad de los ambientes sociales, los cuales debe alinearse con los discursos y géneros textuales escogidos.

El octavo elemento evalúa el diseño semiótico en relación con los procesos de comprensión textual. Profundiza en las implicaciones, tanto discursivas como cognitivas del diseño semiótico en los textos multimodales. Sostiene que el diseño semiótico incide en sus macro y microestructuras y, por lo tanto, en los procesos de comprensión. El diseño rinde cuentas del procesamiento de la información, su organización, su almacenamiento y de cómo es recuperable e interpretada (Kress et al., 2001; Sánchez, Morales, del Pozo y Díaz, 2006).

La evaluación del plano microtextual se enfoca en los usos modales e implica dos aspectos. En primer lugar, el empleo se valora en la medida en que es coherente con las posibilidades de representación del modo mismo; es decir, el uso de los modos respeta su propia naturaleza semiótica. En este punto, entra en juego el concepto de gramática de un modo (Kress y Van Leeuwen, 2001), la cual se entiende como los patrones de organización y combinación de los recursos modales, que definen las posibilidades de representación y las formas en que se puede emplear dentro de una comunidad de creadores de signos. Así, el noveno elemento evalúa el uso modal en función de la gramática: los entornos socioculturales y las reglas, tanto implícitas como explicitas, que rigen su utilización.

En segundo lugar, la evaluación microtextual de los usos modales concierne no solo a la gramática de los modos como tal, sino a su articulación como recursos de significado, en función de géneros textuales y propósitos comunicativos específicos (Kress y Van Leeuwen, 2001; Kress et al, 2001) Para este fin, el décimo y undécimo elemento valoran la alineación entre el empleo de los modos, el género, el discurso, la audiencia meta y las instancias de producción. Esta perspectiva coincide con la lingüística textual, según la cual la producción requiere elaborar el texto desde las unidades inferiores hacia las superiores, a través de las cuales el significado se entreteje, hasta conformar el discurso. 


\section{Competencia 7: Producir textos multimodales en relación con modelos de géneros textuales, en distintos medios}

La tabla 7 sintetiza los elementos que constituyen la competencia, así como las preguntas orientadoras sobre los procesos implicados en el desempeño de cada elemento.

\section{Tabla 7}

Elementos de la competencia 7 y preguntas orientadoras

Elementos de la competencia Preguntas orientadoras

\section{Fase de planificación}

1. Seleccionar un modelo textual para la producción de textos multimodales, según propósitos comunicativos y sus características macro y microtextuales específicas.

2. Crear textos multimodales que no cumplan con las características de un modelo textual, según propósitos comunicativos.

3. Justificar la selección o el descarte de modelos textuales, según sus respectivas características, para la producción de textos multimodales de diversos géneros textuales.

4. Establecer relaciones entre discursos y géneros, en función de los propósitos comunicativos y la audiencia de textos multimodales.

5. Diseñar discursiva y semióticamente los textos multimodales, en concordancia con su entorno sociocultural de producción, circulación y recepción.

\section{Fase de textualización}

6. Emplear los modos semióticos para la creación de textos multimodales, de acuerdo con su propia gramática, los discursos, géneros textuales, los propósitos comunicativos y la audiencia.

7. Utilizar los medios de materialización y distribución de textos multimodales, en relación con el diseño semiótico, el discurso, el género, los propósitos comunicativos y la audiencia.
¿Cuál tipo de texto podría modelar la propia producción textual?

¿El modelo escogido es adecuado para los propósitos comunicativos y la audiencia del texto por producir? ¿Cuáles características a nivel macro y microtextual sugiere el modelo para el propio texto?

¿En qué medida es posible desligarse del modelo textual más cercano para producir un nuevo texto? ¿Cuáles repercusiones en el significado supone separarse de un modelo de género textual? ¿Cuáles características adquiere a nivel macro y microtextual el nuevo texto? ¿Es posible crear el texto sin modelo alguno de referencia? Sin incumplir con un modelo, ¿el texto producido puede destinarse a la misma audiencia? ¿Permite emplear los modos típicos para los tipos de textos más cercanos?

¿Por qué este modelo es pertinente para la construcción del propio texto? ¿Cuáles ventajas posee sobre otros géneros textuales? ¿Cuáles limitaciones supone su selección? ¿Cuáles características macro y microtextuales apoyan su escogencia? ¿Por qué este modelo no es relevante para elaborar el propio texto? ¿Cuáles aspectos no son coherentes con los propios propósitos comunicativos, la audiencia, el contexto comunicacional o sociocultural? ¿Cuáles características macro y microtextuales sugieren descartarlo?

¿Cuáles discursos se desea comunicar? ¿Por qué se quiere comunicarlos? ¿Cuáles tipo(s) de género(s) textual(es) se ajustan para comunicar estos significados? ¿El género permite alcanzar el propósito comunicativo? ¿A quién se destina el discurso? ¿Cuáles características posee la audiencia? ¿La audiencia está familiarizada con este género? ¿El género permite comunicar el discurso para esta audiencia y cumplir, a la vez, el propósito comunicativo?

¿En cuál entorno sociocultural se está produciendo el texto? ¿Hay significados que el entorno motiva/ impide comunicar?

¿Cuáles modos están disponibles en el entorno sociocultural para crear el género específico? Dentro de ellos, ¿cuáles modos son los más aptos para representar los discursos? ¿Cómo se utilizan los modos, normalmente, en la cultura, para el género escogido? ¿Cuáles medios están disponibles en el entorno sociocultural para materializar y distribuir el texto? ¿Cómo suelen emplearse los medios, en la cultura, para el género escogido?

¿Cómo puedo articular los modos? ¿Sé cómo utilizarlos? ¿Requiero desarrollar habilidades para emplearlos? ¿Cuáles aspectos discursivos quiero representar a través de cada modo? ¿A través de su ensamble en conjunto? ¿Se puede representar más de un significado a través de un modo? ¿El género escogido requiere utilizar los modos de una manera determinada? ¿Es posible emplear los modos de otras maneras? ¿Las modalidades son adecuadas para la audiencia? ¿Sus alcances de representación permiten cumplir con los propósitos comunicativos?

¿Sé cómo utilizar los medios para materializar el texto?, ¿distribuir el texto? ¿Existen relaciones entre las formas de materialización y distribución? ¿Los medios de materialización y distribución del texto son coherentes con los modos empleados en el texto? ¿Inciden en los significados discursos representados? ¿Los medios de materialización y distribución del texto son coherentes con el género? ¿Estos medios permiten que el texto alcance a la audiencia? ¿La materialización y circulación del texto, a través de los medios, permiten que cumpla sus propósitos comunicativos? 
8. Aplicar procesos de transformación y transducción de los significados, para la representación de discursos a través de distintos géneros y modalidades.

\section{Fase de revisión}

9. Evaluar la construcción semiótica y discursiva de los textos multimodales, en función de los propósitos del intercambio, la audiencia y el contexto sociocultural.
¿Cómo se pueden definirse los diferentes aspectos discursivos, a través de diversas modalidades? ¿Es posible/necesario trasladar el discurso de un género a otro? ¿Trasladar el discurso implica modificar el diseño semiótico? ¿Requiere emplear otros modos? ¿Otros medios? ¿El discurso mismo -sus significados -se ve modificado por el paso entre géneros? ¿Por el uso de otras modalidades? ¿Por el empleo de otros medios?

¿El diseño obtenido permite comunicar el discurso según el propósito comunicativo? ¿Se ajusta a la audiencia? ¿Cuáles significados discursivos comunican los modos escogidos, una vez articulados en el texto? ¿Son los significados que se desea comunicar? ¿El uso modal es coherente con los géneros y sus propios potenciales de significación? ¿Cuáles aspectos discursivos predominan y cuáles son invisibilizados a través del diseño? ¿El diseño semiótico del texto es claro, coherente con su género, de forma que es interpretado por la audiencia? ¿El diseño semiótico obtenido para el discurso, en este género, es coherente con el entorno sociocultural?

Fuente: Elaboración propia (2020).

Esta última competencia está enfocada en la producción de textos multimodales, según propósitos comunicativos, la audiencia y el contexto sociocultural. Sus elementos se organizan en las etapas de planificación, textualización y revisión (Cassany, 1999), ya que son las sugeridas por el MEP (2017, pp. 194 - 196) y favorecen la implementación de esta propuesta. A las etapas citadas, el artículo suma aspectos de la semiótica social y la lingüística textual, a fin de adaptarlas para la producción de textos multimodales y a las competencias en apartados previos.

Además de organizar la producción en estas tres etapas, la competencia confiere especial relevancia al uso de modelos textuales como estrategia didáctica para orientar la producción, los cuales -por lo demás-, se ajustan a las tres fases de producción previas. Como modelo textual se conciben "unos esquemas cognitivos y textuales, definidos culturalmente, que se aplican dentro de los procesos de escritura y lectura de un texto concreto como respuesta a una intención comunicativa" (Trujillo, 2002, p. 5). Así definidos, los modelos engloban tanto las superestructuras (Van Dijk, 1983) como los llamados géneros discursivos (Swales, 1990); es decir, combinan el carácter cognitivo y el textual (Trujillo, 2002).

Los modelos textuales son importantes para los procesos de enseñanza y aprendizaje por dos motivos principales. En primer lugar, son utilizados en la etapa de planificación textual, como esquemas almacenados en la memoria que favorecen la organización de las ideas, la articulación de la estructura textual, la cohesión y la coherencia. En segundo lugar, la audiencia también emplea estos modelos en los procesos de recepción e interpretación de los textos, para formular predicciones acerca de la organización, el contenido y la relevancia de sus partes constitutivas (Trujillo, 2002).

La planificación textual se define como la etapa del proceso de escritura destinada a tomar conciencia de la situación comunicativa y diseñar un texto a fin de cumplir los propósitos comunicativos. Según se describe, requiere analizar el contexto situacional, generar ideas para la producción a partir de este y elaborar un primer acercamiento (borrador) del texto (Cassany, 1999). A raíz de estas características, en esta fase, se han creado tres elementos relacionados con el uso de los modelos textuales.

El primero de ellos parte de la lingüística textual y sostiene que el desarrollo de las habilidades comunicativas de comprensión y producción ha de fundamentarse en el discurso (Van Dijk, 1993). Esta construcción de los discursos, a su vez, no se realiza de forma aleatoria, sino mediante intercambios comunicativos regulares, cuya repetición termina por estabilizar "tipos", modelos de textos, en función de ciertas interacciones. Así, el primer elemento motiva a seleccionar un modelo textual para la producción, a fin de contar con "un marco de normas y de puntos de referencia para los participantes del intercambio" (Charaudeau, 2006, p.41).

El segundo elemento es diametralmente opuesto al anterior y motiva la creación de textos multimodales sin modelo previo alguno, por lo que su función debe ser entendida en referencia con el primero. 
La relevancia de emplear modelos radica, precisamente, en la relación contractual entre la instancia de producción y recepción que deriva de estos tipos de textos y que garantiza la reciprocidad (Charaudeau, 2006). No obstante, pese la pertinencia de los modelos genéricos, también se considera necesario dotar de libertad creativa al estudiantado para desligarse de estos últimos y explorar posibilidades de significación de originalidad propia.

Dicho de otra manera, el dominio de las convenciones de un género y un modo, por tanto, no implica no solo el conocimiento sobre "lo que se puede hacer", sino también de aquellas limitaciones en el uso y articulaciones que aún no han sido exploradas. Para cumplir esta finalidad, se propone la libertad creadora en la producción de textos, la cual ha sido respaldada por varios estudios que enfatizan la importancia didáctica de la creatividad en los procesos cognitivos de aprendizaje (Craft, Jeffrey y Leibling, 2001; Cropley, 2001).

El tercer elemento está enlazado con los dos anteriores y solicita una argumentación sobre los modelos textuales empleados para la producción textual, así como - de ser el caso - de las razones que motivan la ausencia de ellos. Fundamentalmente, una estrategia metacognitiva en la creación, que enfrenta al estudiantado ante su propio texto y le solicita dar cuenta de él, en términos discursivos, genéricos y semióticos. Permite al creador de texto advertir, seleccionar y atender sus propios procesos cognitivos. Es una herramienta para la autoregulación en el aprendizaje (Tormo, 2017).

El cuarto elemento en la fase de planificación supone alinear el discurso con el género textual y los diversos aspectos de la situación comunicativa, de manera que el texto cumpla con sus propósitos en el marco comunicativo específico. Para tal fin, debe atender el contexto situacional, el asunto o tema (explícito e implícito), las instancias que lo generan y lo reciben, las instancias sobre quién pretende generar sus efectos, así como las relaciones de poder que se despliegan (García, 2016)

En el quinto elemento, la etapa de planificación concierne al nivel del diseño semiótico del texto. Implica analizar los modos y medios con los que cuenta el usuario dentro de un grupo sociocultural, para realizar, materializar y distribuir el discurso en un género textual concreto. En ese sentido, la planificación requiere valorar las restricciones semióticas y socioculturales para articular los discursos, en calidad de fenómenos situados socialmente. También, supone tomar decisiones en relación con los entornos de producción, circulación y recepción textual.

La etapa de textualización consiste en transformar el proyecto planificado en un texto. Para ello, desde un foco lingüístico, es necesario valorar aspectos morfosintácticos; entrelazar las proposiciones en el discurso mediante recursos de cohesión, a fin de garantizar la coherencia textual y, finalmente, producir físicamente el documento (Cassany, 1999). Desde una perspectiva multimodal, la textualización está estrechamente vinculada con las alfabetizaciones múltiples (Cope y Kalantzis, 2009a; 2015; Jewitt, 2008), por cuanto requiere el aprendizaje de una diversidad de formas de representar y comunicar los discursos en forma de textos multimodales, según disciplinas curriculares específicas.

Esta propuesta de competencias concibe la textualización como una oportunidad para la alfabetización múltiple, por cuanto se desliga de la enseñanza exclusiva de los modos lingüísticos y busca el accionar de otros recursos semióticos para la representación y comunicación de los significados, al servicio de géneros y propósitos comunicativos específicos. Así, la textualización supone ejercitarse en el uso de un vasto repertorio de recursos semióticos, en calidad de herramientas de significación y, más ampliamente, para la comunicación a nivel social (Kress, 2010; Cope y Kalantzis, 2015). La ausencia de conocimientos sobre los modos requiere que el creador del texto supla los vacíos para su uso adecuado, es decir, supone un estímulo para su alfabetización.

En la propuesta, se han creado tres elementos de la competencia relacionados con la textualización. El sexto atañe el uso concreto de los modos y medios en la creación de los textos multimodales, y se relaciona con las alfabetizaciones múltiples. El empleo de los modos para la creación textual requiere el dominio 
de su propia gramática, a fin de utilizar apropiadamente su potencial de significación y articularlos con otras modalidades presentes en el texto (Cope y Kalantzis, 2009a). Los usos modales deben procurar la coherencia con el género y el discurso, es decir, los modos deben emplearse de forma que se cumpla el propósito comunicativo del texto en función de su audiencia meta.

Por otra parte, la textualización también conlleva el uso de medios para materializar y distribuir los textos, de manera que el usuario requiere conocimientos para aprovechar, de forma adecuada, sus posibilidades. En el séptimo elemento, se debe considerar que los propios alcances de representación de cada modo favorecen el uso de ciertos medios más adecuados para su materialización, debido a su naturaleza semiótica (Kress, 2010). Así, el uso de los medios también debe estar de acuerdo con los modos empleados y, en última instancia, con el diseño semiótico del texto. También, debe estar acorde con las posibilidades de empleo ofrecidas en su entorno sociocultural.

El octavo elemento parte del hecho de que, a pesar de llevar a cabo una frase de planificación previa, el proceso de textualización puede no ser uno solo o perfecto, sino constante, de prueba y error, con múltiples ajustes. Aún más, ante las sucesivas versiones del texto, el creador podría reconsiderar y enfrentar cambios entre las modalidades, entre géneros o aspectos discursivos, que lo llevarían a poner en práctica procesos de transformación y transducción semiótica (Kress, 2010). De ahí la importancia de incorporar un elemento dentro de esta competencia que permita implementar los principios señalados.

La etapa de revisión consiste en evaluar los textos producidos, lo cual requiere dos pasos fundamentales: una valoración de la construcción genérica y discursiva, a nivel macro y microtextual, y la corrección de las potenciales limitantes encontradas en el texto, tras la primera valoración (Cassany, 1999). En la propuesta, la revisión adopta un tratamiento particular en el elemento de la competencia número nueve, el cual se decidió concatenar con la competencia previa de evaluación. Es decir, se sugiere al profesorado que, alcanzada la fase de revisión de los textos multimodales, se remita a la competencia previa y aplique los elementos ahí descritos.

La decisión obedece a varias motivaciones. En primer lugar, los procesos de revisión constituyen, en sí mismos, una forma de evaluación de los textos producidos (Cassany, 1999). Por tal motivo, requieren desarrollar un conjunto de criterios y pautas que permitan valorar adecuadamente los textos creados. Por tanto, se considera que la competencia anterior desglosa, de forma detallada, una valoración crítica de los textos multimodales, según sus propósitos comunicativos, la audiencia y el contexto sociocultural, por lo que es susceptible de utilizarse en esta competencia final para la revisión.

Por otra parte, los elementos de la competencia de evaluación están construidos de forma amplia y sistémica, a fin de abordar las múltiples relaciones entre discursos, géneros, modos y medios en textos multimodales. Así, se consideran herramientas claras y didácticas para guiar el proceso de revisión. Finalmente, la competencia previa muestra la ventaja de ubicarse antes de la competencia de producción. Siguiendo el orden propuesto, se espera que tanto el profesorado como el estudiantado se familiaricen primero con procesos de valoración de textos ajenos, para luego, al llegar a este nivel, aplicarlos para la revisión de los propios.

\section{SÍNTESIS Y REFLEXIONES FINALES}

En relación con el plan de estudios costarricense para la asignatura de español (MEP, 2017), la propuesta sostiene la necesidad de incorporar una dimensión semiótica a la competencia comunicativa (Lomas, 1999; citado en MEP, 2017), ante la presencia, en dicho documento, de géneros textuales con complejas características multimodales y los objetivos de análisis, interpretación y producción multimodal que se persiguen. Su incorporación es necesaria en el marco de la transición pedagógica contemporánea (Walsh, 2010), en la que tanto la política educativa como los planes de estudio deben adaptarse a la amplia presencia de medios digitales y textos multimodales asociados. 
Las competencias semióticas aquí esbozadas parten de la noción de género discursivos, el contexto situacional y sociocultural, para generar procesos de aproximación, caracterización, análisis, explicación, evaluación y producción de textos multimodales. Así, procuran desarrollar habilidades para una alfabetización crítica, que permitan al profesorado y estudiantado descubrir las relaciones entre los propósitos comunicativos, las audiencias, los discursos y la composición semiótica, tanto en textos multimodales dados, como creados por autoría propia (Anstey y Bull 2006; Cooper et al., 2013; Walsh 2010).

La ejercitación de estas competencias, a su vez, pretende apoyar el desarrollo de habilidades para la comprensión de textos de naturaleza multimodal. En ese sentido, la propuesta es complementaria a otros estudios, en el ámbito internacional, que indagan en las particularidades en la lectura de textos multimodales, que emergen de su configuración semiótica (Parodi y Julio, 2017); ofrecen categorías analíticas que posibilitan su lectura y rutas para apoyar los procesos pedagógicos por aplicar a estos textos (García-Dussán, 2015), o programas de alfabetización visual crítica de textos multimodales para la educación en lenguas (Farías y Araya, 2014).

La comprensión de lectura, en textos multimodales, exige el desarrollo de competencias que permitan al estudiantado (Farías y Araya, 2014, p. 98), "reflexionar de manera crítica sobre el poder de los textos multimodales y comprender que la construcción de textos no es una actividad neutral, asumir una conciencia retórica como productores y lectores de textos, y desarrollar la participación activa como comunicadores y no solo como receptores pasivos de mensajes mediáticos". Para alcanzar estos fines, las competencias 1 - 3 se enfocan en el análisis gráfico - estructural del texto (García-Dussán, 2015), cuyo objetivo es caracterizar el diseño semiótico. Las competencias de la 3 - 5 se enfocan en los procesos sociosemióticos, intra, extra e intertextuales (GarcíaDussán, 2015); es decir, en la comprensión e interpretación de los significados representados.

El estudio de la comprensión lectora de textos multimodales constituye un ámbito especialmente novedoso y poco explorado en Costa Rica. En estas circunstancias, nace el proyecto de investigación Desarrollo de comprensión lectora de textos multimodales en secundaria, financiado por el Espacio de Estudios Avanzados de la Universidad de Costa Rica, UCREA. Su interés radica, precisamente, en proponer modelos de comprensión lectora de textos multimodales y estrategias para este fin, susceptibles de aplicarse en la interacción de aula, lo cual constituye nuevas líneas de reflexión en el campo de las Ciencias de la Educación, en función los textos multimodales, los procesos de enseñanza y aprendizaje.

Esta propuesta junto con los estudios en desarrollo -en el marco del proyecto anterior- constituyen líneas investigativas para favorecer el abordaje de textos multimodales por parte del profesorado y del estudiantado, en cumplimiento de los objetivos curriculares del MEP. Sus esfuerzos se han pensado para estar a la altura del desarrollo internacional de otras propuestas pedagógicas que procuran afianzar el desarrollo de las alfabetizaciones múltiples y motivar cambios en los procesos didácticos y curriculares desde una perspectiva multimodal (Anstey y Bull 2006; Cope y Kalantzis, 2009b; Blanchard y Farstrup 2011; Cooper et al., 2013; Reid and Comber 2004; Walsh 2010).

Nota: El Modelo de Análisis de Géneros Multimodales (MAGEM) es creado dentro del marco del proyecto de investigación N. ${ }^{\circ}$ 021-B9-781 Desarrollo de comprensión lectora de textos multimodales en secundaria, financiado por el Espacio de Estudios Avanzados de la Universidad de Costa Rica, UCREA.

\section{REFERENCIAS}

Albers, P., y Harste, J. C. (2007). The arts, new literacies, and multimodality. English Education, 40(1), 6-20. Recuperado de: https://www.jstor.org/stable/40173265

Anstey, M. y Bull, G. (2006). Teaching and learning multiliteracies: Changing times, changing literacies. Delaware, USA: International Reading Association. 
Bateman, J. (2008). Multimodality and genre: A foundation for the systematic analysis of multimodal documents. doi, https://doi.org/10.1057/9780230582323

Bezemer, J., y Kress, G. (2008). Writing in multimodal texts a social semiotic account of designs for learning. Written communication, 25(2), 166-195, doi: https://doi.org/10.1177/0741088307313177

Blanchard, J.S. y Farstrup A.E. (2011). Technologies, digital media, and reading instruction. En Samuels, S.J., y Farstrup, A.E. (2011) What Research has to say about reading instruction. Delaware, USA: International Reading Association.

Cassany, D. (1999). Construir la escritura. Madrid, España: Grupo Planeta.

Charaudeau, P. (2004). La problemática de los géneros: De la situación a la construcción textual. Revista signos, 37(56), 23-39. doi, https://dx.doi.org/10.4067/S0718-09342004005600003

Charaudeau, P. (2006). El contrato de comunicación en una perspectiva lingüística: Normas psicosociales y normas discursivas. Opción, 22(49), 38-54. Recuperado de: https://www.redalyc.org/articulo. oa?id=310/31004904

Cooper, N., Lockyer, L., y Brown, I. (2013). Developing multiliteracies in a technology-mediated environment, Educational Media International, 50(2) 93-107. doi, https://doi.org/10.1080/09523987.201 3.795350

Cope, B., y Kalantzis, M. (2009a) "Multiliteracies": New Literacies, New Learning. Pedagogies: An International Journal, 4(3), 164-195. doi, https://doi.org/10.1080/15544800903076044

Cope, B., y Kalantzis, M. (2009b). A Grammar of Multimodality. International Journal of Learning, 16(2), 361-425. doi, https://doi.org/10.18848/1447-9494/CGP/v16i02/46137

Cope, B., y Kalantzis, M. (2015). The things you do to know: An introduction to the pedagogy of multiliteracies. En A pedagogy of multiliteracies (pp. 1-36). London, UK: Palgrave Macmillan.

Craft, A., Jeffrey, B., y Leibling, M. (Eds.). (2001). Creativity in education. London, UK: AyC Black.

Cropley, A. J. (2001). Creativity in education y learning: A guide for teachers and educators. London, UK: Psychology Press.

Farías, M. \& Araya, C. (2014). Alfabetización visual crítica y educación en lengua materna: estrategias metacognitivas en la comprensión lectora de textos multimodales. Colombian Applied Linguistics Journal, 16(1), 93-104, doi: https://doi.org/10.14483/udistrital.jour.calj.2014.1.a08

Flores, C. (2019). Modos semióticos y géneros en la enseñanza de lengua y literatura en secundaria: un estudio de caso (Tesis de maestría sin publicar). Universidad de Costa Rica, Costa Rica.

García, F. (2016). El análisis del discurso como práctica social. Glosa, 4(7), 19-26. Recuperado de: http:// www.revistaglosa.com/articulos7

Gibbons, A. (2012). Multimodality, cognition, and experimental literature. London, UK: Routledge.

Jewitt, C. (2008). Multimodality and literacy in school classrooms. Review of Research in Education, 32(1), 241-267. doi, https://doi.org/10.3102/0091732X07310586

Kress, G. (2003). Literacy in the New Media Age. London, UK: Routledge.

Kress, G. (2005). Gains and losses: New forms of texts, knowledge, and learning. Computers and composition, 22(1), 5-22. doi, https://doi.org/10.1016/j.compcom.2004.12.004

Kress, G. (2010). Multimodality: A social semiotic approach to contemporary communication. London, UK: Routledge.

Kress, G., y van Leeuwen, T. (2001). Multimodal discourse: The modes and media of contemporary communication. New York, USA: Hodder Arnold. 
Kress, G., Jewitt, C., Ogborn, J., y Tsatsarelis, C. (2001). Multimodal teaching and learning: the rhetorics of the science classroom. London, UK: Continuum.

Lemke, J. (1998). Multiplying meaning: Visual and verbal semiotics in scientific text. En Martin, J \& Veel, R. (Eds.). Reading science, pp. 87-113. London, UK: Routledge.

Livingstone, S., and Das, R. (2013) Interpretation/Reception. In P. Moy (Ed.), Oxford Bibliographies Online: Communication. Oxford, UK: Oxford University Press. doi: https://doi.org/10.1093/ obo/9780199756841-0134

Lomas, C. (1999). Cómo enseñar a hacer cosas con las palabras. Vol I. Barcelona, España: Paidós.

Martinec, R., y Salway, A. (2005). A system for image-text relations in new (and old) media. Visual Communication, 4(3), 337-371, doi: https://doi.org/10.1177/1470357205055928

Ministerio de Educación Pública. (2017). Programa de estudio de español: Comunicación y comprensión lectora. Tercer ciclo y educación diversificada. Recuperado de: https://www.mep.go.cr/ programa-estudio/espanol

Parodi, G., \& Julio, C. (2017). No solo existen palabras en los textos escritos: algunas teorías y modelos de comprensión de textos multimodales o multisemióticos. Investigaciones Sobre Lectura, 8, 27-48. Recuperado de: https://dialnet.unirioja.es/servlet/articulo?codigo=6103194

Ramírez, J., y Solano, S. (2016). Análisis e interpretación de textos literarios. Recuperado de: http://uprid2.up.ac.pa:8080/xmlui/handle/123456789/1465

Reid, J., y Comber, B. (2004). Theoretical perspectives in early literacy education: Implications for practice. En Makin, L. y Jones Diaz, C. (Eds.). (2004) Literacies in early childhood: Changing views challenging practice. Marrickville, USA: Maclennan and Petty.

Sánchez, R., Morales, E., del Pozo, E., y Díaz, M. (2006). La aceleración de los procesos de producción y circulación de información. Revista Iberoamericana de Educación, 37(4), 1-8. Recuperado de: https:// bit.ly/31fVgg5

Santander, P. (2011). Por qué y cómo hacer análisis del discurso. Cinta Moebio, 41 207-224. Recuperado de: http://www.redalyc.org/pdf/101/10119954006.pdf

Swales, J. (1990). Genre Analysis: English in academic and research settings. Cambridge, UK: University Press.

Talbert, R. (2019). The flipped classroom. Re-thinking Bloom's Taxonomy for flipped learning design. Recuperado de: http://rtalbert.org/re-thinking-blooms-taxonomy-for-flipped-learning-design/

Tobón, S. (2005). Formación basada en competencias: Pensamiento complejo, diseño curricular y didáctica. Bogotá, Colombia: Ecoe ediciones.

Tormo, E. (2017). Reflexión metalingüística en el proceso de composición escrita cooperativa en el marco de una secuencia didáctica. (Tesis doctoral). Universidad Autónoma de Barcelona, Barcelona. Recuperado de: https://www.tdx.cat/handle/10803/458620\#page=1

Trujillo, F. (2002). Los modelos textuales en la enseñanza de la escritura y la lectura. Eúphoros, (4), 11-22. Recuperado de: https://dialnet.unirioja.es/servlet/articulo?codigo=1183007

Unsworth, L. (2006). Towards a metalanguage for multiliteracies education: Describing the meaningmaking resources of language-image interaction. English Teaching: Practice and Critique, 5(1), 55-76. Recuperado de: https://scinapse.io/papers/2120161421

Van Dijk, T. (1983). La ciencia del texto. Barcelona, España: Paidós.

Van Dijk, T. A. (1980a). Macroestructures. An Interdisciplinary Study of Global Structures in Discourse. Cognitions and Interaction. New Jersey, USA: Lawrence Erlbaum.

Van Dijk, T. A. (1980b). Estructuras y funciones del discurso. Madrid, España: Siglo XXI. 
Van Dijk, T. A. (1993). Principles of critical discourse analysis. Discourse and society, 4(2), 249-283. doi, https://doi.org/10.1177/0957926593004002006

Van Leeuwen, T. (2005). Introducing social semiotics. London, UK: Psychology Press.

Verde, S. (2015). El desarrollo de la competencia pragmática: aproximación al estudio del procesamiento pragmático del lenguaje (Tesis doctoral sin publicar). Universidad de Salamanca, España. Doi, https://doi.org/10.14201/gredos.128306

Walsh, M. (2010). Multimodal literacy: what does it mean for classroom practice? Australian Journal of Language and Literacy, 33(3), 211-239. Recuperado de: http://www.alea.edu.au/documents/item/63 